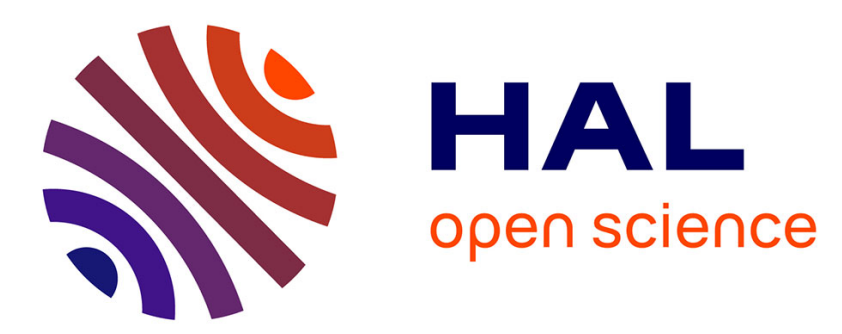

\title{
Polyp follow-Up in an Intelligent Wireless Capsule Endoscopy
}

Orlando Chuquimia, Andrea Pinna, Xavier Dray, Bertrand Granado

\section{To cite this version:}

Orlando Chuquimia, Andrea Pinna, Xavier Dray, Bertrand Granado. Polyp follow-Up in an Intelligent Wireless Capsule Endoscopy. 2019 IEEE Biomedical Circuits and Systems Conference (BioCAS), Oct 2019, Nara, Japan. pp.1-4, 10.1109/BIOCAS.2019.8919016 . hal-02495524

\section{HAL Id: hal-02495524 https://hal.science/hal-02495524}

Submitted on 2 Mar 2020

HAL is a multi-disciplinary open access archive for the deposit and dissemination of scientific research documents, whether they are published or not. The documents may come from teaching and research institutions in France or abroad, or from public or private research centers.
L'archive ouverte pluridisciplinaire HAL, est destinée au dépôt et à la diffusion de documents scientifiques de niveau recherche, publiés ou non, émanant des établissements d'enseignement et de recherche français ou étrangers, des laboratoires publics ou privés. 


\title{
Polyp follow-Up in an Intelligent Wireless Capsule Endoscopy
}

\author{
Orlando Chuquimia ${ }^{1}$, Andrea Pinna ${ }^{1}$, Xavier Dray $^{2}$, Bertrand Granado $^{1}$
}

\begin{abstract}
In this paper, an image processing to detect polyps in an intelligent Wireless Capsule Endoscopy (WCE) is presented. This processing will be integrated into the WCE. It is a new screening method to detect colorectal cancer (CRC). A motion estimation algorithm is used to follow a detected polyp and improve the pre-processing of our detection chain. With our methodology, the polyp detection rate is improved by up to $40 \%$ from $53 \%$ to 93.7\%. The improved detection rate was validated with a large database of 20 video-colonoscopies (18,910 images).
\end{abstract}

\section{INTRODUCTION}

CRC is the second highest cause of death by cancer worldwide with 880,792 deaths in 2018 and a mortality rate of $47.6 \% .95 \%$ of CRC cases begin with the presence of a growth on the inner lining of the colon or the rectum, called a polyp. Multiple types of polyps exist; among them, adenoma polyps, which can degenerate into CRC. CRC is treatable in $90 \%$ of the cases if it is detected early enough [1].

Today, imaging is the modality used to analyze the colon and find polyps. A colonoscopy is the procedure used for screening, diagnosis, and therapy in the gastrointestinal tract. However, it can be painful, traumatic and poorly tolerated by patients. The colonoscopy is invasive and usually requires anesthesia, a specialist and a controlled environment. Furthermore, the colonoscopy does not allow the visibility of all the regions near the colon. Other methods exist, such as the Colorectal Tomography (CT) and the WCE. CT is noninvasive. However, this method cannot detect polyps smaller than $1 \mathrm{~cm}$ and exposes the patient to radiation. WCE is less invasive; it is a simple pill that the patient swallows and that transmits images of the gastrointestinal tract via a Radio Frequency communication through the body. The video feedback is then uploaded to a workstation where a specialist can review and examine these images to detect any gastroenterological pathology. The available WCE PillCam Colon 2 [2] has a length of $32.3 \mathrm{~mm}$, a diameter of $11.6 \mathrm{~mm}$, a battery life of 10 hours, a resolution of 256x256 pixels and an image sampling rate of approximately 2 to 4 frames per second. Ten hours is not sufficient to inspect the total intestinal tract. In addition, WCE has a low image resolution compared to a standard endoscope used in a colonoscopy, that acquires an image with a resolution of $1920 \times 1080$ pixels. A WCE generates between 144,000 and 1,260,000 images (most of these images do not contain polyps or any gastroenterological pathology). Visual analysis of this large number of images with low resolution makes the examination difficult and timeconsuming for the gastroenterologist.

\footnotetext{
${ }^{1}$ LIP6, CNRS UMR 7606, Sorbonne Université, Paris, France.

${ }^{2}$ APHP, Hôpital Saint-Antoine, Sorbonne Université, Paris, France.
}

How can we create a new tool without the side effects and limitations of both colonoscopy and WCE, to offer a powerful screening tool and to reduce the CRC mortality rate?

We propose a new paradigm of WCE: an intelligent WCE (iWCE). Its novelty is to integrate processing capabilities directly in the capsule to automatically recognize a polyp. Our iWCE integrates a high-resolution imager as an endoscope (1920x1080 pixels) and an image processing chain to detect suspicious lesions. It solely transmits images with suspected gastroenterological pathologies. This method will reduce energy consumption and the number of images to be analyzed. The processing is defined to be integrated taking into account multiple constraints; a square surface area constraint of 8 by $8 \mathrm{~mm}^{2}$ (due to $11.6 \mathrm{~mm}$ capsule diameter), a real-time constraint of 25 frames per second, and a power consumption constraint of a battery life greater than 12 hours. Our iWCE is a new screening method and a minimally invasive diagnostic tool that will overcome the current barriers that limit the use of WCE. Specialists will thus take clinical decisions in a time-effective manner without analyzing more than a hundred thousand images.

As this iWCE is a new approach, there are few existing works. However, scientific works containing off-line analysis of the images from endoscope or WCE detecting polyp lesions can be found. A review of some of the computer-assisted polyp detection methods can be found in [3].

These methods can be divided into three groups:

1) Hand-crafted approaches that exploit low-level image processing algorithms to detect region candidates with a polyp, inlcuding intensity valley [3] or Hessian filters [4].

2) Machine learning approaches based on classifiers that can be a Convolutional Neural Network (CNN) to detect polyp lesions as in [5].

3) Hybrid approaches which combine both methodologies. Works in [6] adopt this strategy with co-occurrence matrix and local binary patterns as Hand-craft processing. Classifiers can be, for example, Multi Layer Perceptrons or Adaboost.

All these methods run on an external computer and contribute to helping the physician in his diagnosis, but they are not useful for our purpose. They are not suitable to be integrated into a WCE and they do not take into account certain constraints, such as real-time execution, form factor of the pill and energy consumption. In particular, CNN methods use several million of synapses and neurons, which cannot be integrated in a 8 by $8 \mathrm{~mm}^{2}$ chip in a pill, with a power consumption limitation of $158.1 \mathrm{~mW}$ [2].

In our previous works, a hybrid approach based on an image processing chain and fuzzy trees used as classifiers [7] was 
proposed. This approach was used to detect polyps and it was designed to be integrated in a WCE. Information about the integration can be found in the article [8]. The first step in the image processing chain is to extract regions of interest (ROI) from an image. These ROIs correspond to parts of the image where the texture features could indicate the presence of polyps. In the second step, the ROIs are classified using fuzzy trees as the machine learning algorithm.

To study the performance of our approach, we used a public database named ASU-Mayo Clinic Colonoscopy Database, which was introduced during the EndoVisSub2015-GIANA challenge at MICCAI 2015 [3]. It is composed of 18,996 images (4,278 images containing a polyp) and their respective ground-truths. The ground-truth is a binary-image that indicates the position of the polyp in the image. The images come from 20 video-colonoscopies in which 10 display a unique polyp at multiple scales and from different viewing angles.

We have manually extracted the ROIs based on the groundtruth of this database. Next, we trained the fuzzy tree to obtain two classes, a class 1 for the presence of a polyp and a class 0 for the absence of a polyp. We use $70 \%$ of the images to learn and $30 \%$ of the images to test.

To measure the performance, we have computed two indicators at ROI-level:

- The sensitivity $=\frac{T P}{T P+F N}$

- The specificity $=\frac{T N}{T N+F P}$

with the parameters of the equations defined as:

- TP: a ROI of class 1 that contains a polyp.

- FP: a ROI of class 1 that does not contain a polyp.

- TN: a ROI of class0 that does not contain a polyp.

- FN: a ROI of class0 that contains a polyp.

We have obtained a sensitivity of $93 \%$ and a specificity of $91 \%$.

Although the results are good, a bias is present: the classification results are for manual ROI extractions. The extraction of the ROI by an image processing chain is a crucial part. By giving specific features from the image to the machine learning algorithm, it has a clear impact on the algorithm performance [2].

In this article, we analyze our image processing chain that delivers features from the image to the fuzzy trees. We make a proposition to enhance its performance and finally, we evaluate the performance to come to a conclusion.

\section{EVAlUATION OF THE IMAGE PROCESSING CHAIN}

In this section, we provide a description of our first hybrid approach described in [7], which includes three steps.

1) extraction step: before extracting a ROI, we convert each image from an RGB model to a brightness model. This preserves the texture information and provides a better degree of integrability than an image color processing containing three channels. Then, we realize an edge detection into the image by applying a $3 \times 3$ median noise filter and a Canny filter. Finally, we use a Hough Transform to detect circular or elliptical shapes.
Each circle or ellipse becomes a ROI (see Fig. 1). We chose the Hough Transform because a polyp circular or elliptical shape. It is also possible to integrate it in realtime in a WCE [9].

2) description step: we realize a texture analysis of the ROIs and extract 26 texture and luminosity descriptors using co-occurrence matrix algorithm [10]. Texture and luminosity are important descriptors to recognize and identify polyps [11].

3) classification step: in this step, we use the fuzzy trees to classify each image.

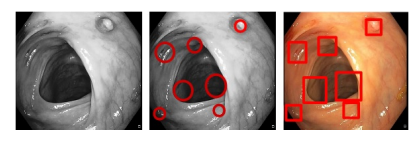

Fig. 1. Extraction step

Here, the image pre-processing part is composed by the two steps; the extraction step and the description step. To evaluate our pre-processing part, we measure the detection rate of the global processing which includes the three steps mentioned above; extraction, description, and classification. The measurements were made at two levels: at ROI-level and at image-level. At ROI-level, a ROI containing a polyp is labeled as class 1 and a ROI without a polyp is classified as class0 [7]. At image-level, an image containing at least one ROI of class 1 is classified as class 1 . Otherwise, it is classified as class 0 .

TABLE I

PERFORMANCES OF THE GLOBAL PROCESSING

\begin{tabular}{|c|c|c|c|c|}
\hline Videos & \multicolumn{2}{|c|}{ ROI level } & \multicolumn{2}{c|}{ Image level } \\
\hline 20 & sensitivity & Spec. & sensitivity & Spec. \\
\hline Results & $29.9 \%$ & $95.2 \%$ & $53.2 \%$ & $73 \%$ \\
\hline
\end{tabular}

The results are shown in Table I. We notice that at the ROIlevel, the sensitivity is only $30 \%$ and at the image-level, the sensitivity is $53 \%$ and the specificity is $73 \%$. This shows the impact of the image pre-processing part. At ROI-level, the performance of the classification step falls from 93\% [7] to $30 \%$. At image-level, the sensitivity and specificity are low.

The reason for this must be examined. In the manual extraction, the ROIs contain the entire polyp according to the ground truth. Furthermore, all the polyps are contained in a ROI. In the case of automatic extraction via image processing, the ROIs do not seem to contain an entire polyp and not all polyps are in a ROI.

We have conducted a deep analysis and determined that by using the proposed image processing, $70 \%$ of polyps were extracted in at least one ROI. Also, we have shown that polyps were not always extracted completely. This impacts the sensitivity performance of our classifier that was trained to recognize ROIs that contain an entire polyp.

We have measured the percentage of polyps contained in the ROIs and obtained the following results:

- $11.5 \%$ contain between $90-100 \%$ of a polyp

- $31.6 \%$ contain between $50-90 \%$ of a polyp

- $56.9 \%$ contain between $1-50 \%$ of a polyp 


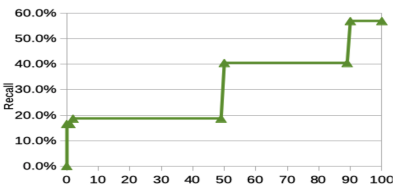

Fig. 2. Sensitivity according the percentage of polyp contained in ROI.

We have observed that a large amount of the ROIs does not contain an entire polyp and only $43 \%$ of the ROIs contain at least $50 \%$ of a polyp. We then measured the sensitivity indicator for each of these three cases. The results are visible in Figure 2.

As expected, a higher sensitivity is associated to ROIs containing higher percentages of the polyp. We need to improve the image processing to extract ROIs containing a high percentage of the polyp.

Additionally, we have analyzed the performances of the global processing at the image-level. At this level, in a first approach, we use a simple rule: an image containing at least one ROI classified as class 1 is classified as class 1 ; all other images are classified as class 0 . The specificity in this case is equal to the probability that all $n$ ROIs are classified as class 0 ; it is a binomial probability equal to:

$$
\text { Specificity }_{\text {Imglevel }}=P_{\left(\text {class }_{R O I}=0\right)}^{n}=\text { Specificity }_{\text {ROI level }}^{n}
$$

The number of ROIs of class 0 in an image varies from 5 to 13 , which could decrease the sensitivity from $78 \%$ to $53 \%$. We measure a decrease of $73 \%$. We notice that the rule used to define the image class is not efficient.

To solve these problems, we propose a new approach for polyp detection based on gastroenterologists' expertise.

\section{Proposed Method}

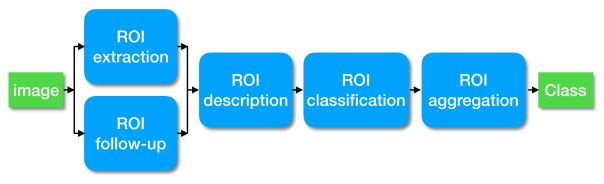

Fig. 3. Modification of our former hybrid approach with now five steps.

Our method is inspired by the gastroenterologists' psychovisual methodology when they are performing a colonoscopy. They select the ROIs inside the gastrointestinal tract based on shape features. Once they select a ROI, they move the endoscope and follow the ROI to include a more accurate analysis and to detect the presence of an existing polyp. Based on this methodology, we modified our hybrid approach to have five steps (see in Figure 3). These steps are the following:

1) extraction step: the same processing as previously mentioned is used.

2) follow-up step: if an ROI was classified as class 1 in the previous image, we use a motion estimation method to determine where the ROI should be in the current image and create a related ROI.

3) description step: the same processing as previously mentioned is used.
4) classification step: the same processing as previously mentioned is used.

5) aggregation step: Images' class is defined by an aggregation of all the ROIs of class 1 in the last images.

In brief:

- We have added a follow-up step. The goal of this step is to improve the extraction of the ROIs that contain a high percentage of a polyp. In the case where a ROI is classified as class 1 in image $I_{n-1}$, we use its location in the image $I_{n-1}$ to estimate its location in the image $I_{n}$. We then place a ROI on this location. This new ROI is sent to the description step. In the follow-up step, the temporal depth is denoted $m$ to analyze the images from $I_{n-1}$ to $I_{n-m}$. In this article, we have used $m=1,2$ and 3 .

- We execute the extraction and the follow-up steps in parallel.

- We have added an aggregation step to determine the image's class by exploiting spatio-temporal information. An image will be considered as class 1 if there is at least one ROI of class 1 that was class 1 on the last $k$ images. In our case, we use $k=3$.

With the follow-up step, we expect to increase the number of ROIs that contain more than $50 \%$ of a polyp by estimating their location in the next image.

With the aggregation step, we expect to increase the specificity at the image level.

In the next subsection, we describe the ROI follow-up step in detail.

\section{A. Follow-up step}

To follow a ROI validated as class 1 , we apply a motion estimation using a block matching algorithm described in Figure 4. Each ROI validated as class 1 in the last image $I_{n-1}$ is considered as a block $B_{p, q}$ of size $P * Q$. For all the pixels in the block, a motion vector is computed.

The motion estimation is performed by computing a similarity measurement between $I_{n-1}\left(B_{p, q}\right)$ and $I_{n}\left(B_{p-i, q-j}\right)$ using the intensity standard variation $\operatorname{Var}(i, j)$ (equation 1). Here, we refer to the vector $I_{n-1}\left(B_{p, q}\right)$ of the image $I_{n-1}$ in the block $B_{p, q}$ as: $I_{n-1}\left(B_{p, q}\right)=\left[I_{n-1}(p, q), \ldots, I_{n-1}(p+P-1, q+Q-1)\right]^{T}$.

$$
\operatorname{Var}(i, j)=\sqrt{\frac{\sum_{\forall_{p, q \in[B x P]}\left[I_{n-1}\left(B_{p, q}\right)-I_{n}\left(B_{p-i, q-j}\right)\right]^{2}}}{B * P}}
$$

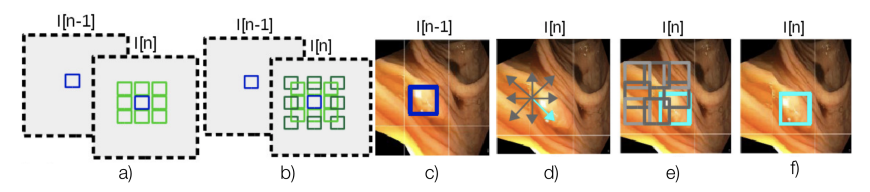

Fig. 4. For a depth=1, a) Block matching with 1 neighborhood. b) Block matching with 2 neighborhoods. c) ROI validated in the image $I_{n-1}$. d) 8 candidate motion vectors. e) 8 candidate blocks displaced by the candidate motion vectors. f) Candidate block having the lowest standard variation.

As an example, in the image $I_{n}$ of Figure 4.a, the block $B$ is displaced from the initial position $(p, q)$ to $(p-i, q-j)$. This 
motion corresponds to a motion vector $\vec{V}=(i, j)$. We identify this vector among 8 candidate motion vectors. To enhance the motion vector identification, we compute the candidate vector motion with different depths; in Figure 4 we see an example with a depth of 1 (a) and a depth of 2 (b). We can have a maximum depth of 10 .

The motion vector $\vec{V}=(i, j)$ will be the candidate motion vector where $\operatorname{Var}(i, j)$ is minimum. The followed ROI in the image $I_{n}$ will be the validated ROI in the image $I_{n-1}$ (see Figure 4) displaced by motion vector $\vec{V}=(i, j)$.

With this technique, we can follow the ROIs where a polyp was detected in the image $I_{n-1}$. Furthermore, we can increase the temporal depth of the motion estimation to follow the ROIs where a polyp was detected in the images $I_{n-1}, I_{n-2}, \ldots, I_{n-m}$.

\section{EXPERIMENTAL RESULTS}

We have evaluated our modified hybrid approach with a temporal depth of $m=0,1,2$ and 3. Additionally, we have evaluated the detection of the polyps at the ROI-level and the image-level (see Table II). In Table II, we notice that adding the aggregation step increases the specificity from $73 \%$ to $83 \%$ if a temporal depth of $m=0$ is used. Furthermore, for a temporal depth of $m=3$, we notice that the sensitivity increases from $30 \%$ to $57 \%$ at ROI-level and from 53\% to $93.7 \%$ at image-level. The performance has increased by $40 \%$ compared to our previous approach while using processing that is compatible with integration inside an iWCE. If we analyze the specificity, we observe an important decrease at the ROIlevel but a limited one of $10 \%$, at the image-level. This can also be considered as a good result.

In addition, we have analyzed the ROIs and showed that the follow-up step improves the extraction of the ROIs with a larger part of the polyps:

- $15.2 \%$ of ROIs contain between $90-100 \%$ of a polyp,

- $40.4 \%$ of ROIs contain between $50-90 \%$ of a polyp,

- $44.4 \%$ of ROIs contain between $1-50 \%$ of a polyp,

TABLE II

PERFORMANCES OF THE MODIFIED HYBRID APPROACH

\begin{tabular}{|c|c|c|c|c|}
\hline & \multicolumn{2}{|c|}{ ROI-level } & \multicolumn{2}{c|}{ Image-level } \\
\hline Temporal depth & sensitivity & Spec. & sensitivity & Spec. \\
\hline $\mathrm{m}=0$ & $33,48 \%$ & $93.1 \%$ & $66.7 \%$ & $82.8 \%$ \\
\hline $\mathrm{m}=1$ & $53.8 \%$ & $86.0 \%$ & $93.2 \%$ & $65.0 \%$ \\
\hline $\mathrm{m}=2$ & $56.8 \%$ & $84.0 \%$ & $93.3 \%$ & $63.6 \%$ \\
\hline $\mathrm{m}=3$ & $57.1 \%$ & $83.1 \%$ & $93.7 \%$ & $62.0 \%$ \\
\hline
\end{tabular}

These results show that our goals have been obtained using our new method. The amount of ROI identified that contains at least $50 \%$ of a polyp increased from $43 \%$ to $56 \%$ by using a simple Block Matching technique. Therefore, taking into account the spatiotemporal information by a ROI follow-up approach increases the performance of the detection.

By using the spatiotemporal aggregation, we increase the specificity and obtain a high score of $\mathbf{9 3 . 7 \%}$ for the sensitivity at the image-level.

\section{CONCLUSIONS}

In this paper, we have presented a hybrid approach to detect polyps that could be integrated in an iWCE [8]. This chain comes from the gastroenterologists' psychovisual methodology. The idea is to follow an extracted ROI using a simple motion estimation algorithm. With our proposition, we show an increase of the sensitivity from 53\% to $93.7 \%$ at image level, validated on a large database of 18,910 images.

Follow-up is based on the analysis of the video stream and not only a per image analysis. Our results show that considering the spatiotemporal location of a polyp on a segment of video can improve the performance of the detection. The next work will be focused on a deep analysis of the spatiotemporal information and the membership degree of a ROI containing a polyp on a segment of the video. In addition, we will consider fuzzy trees to recognize ROIs with non-centered polyp to improve the detection rate. ${ }^{1}$

\section{REFERENCES}

[1] J. Ferlay, I. Soerjomataram, R. Dikshit, S. Eser, C. Mathers, M. Rebelo, D. M. Parkin, D. Forman, and F. Bray, "Cancer incidence and mortality worldwide: sources, methods and major patterns in globocan 2012," International journal of cancer, vol. 136, no. 5, pp. E359-E386, 2015.

[2] A. Karargyris and N. Bourbakis, "Detection of small bowel polyps and ulcers in wireless capsule endoscopy videos," IEEE transactions on Biomedical Engineering, vol. 58, no. 10, pp. 2777-2786, 2011.

[3] J. Bernal, N. Tajkbaksh, F. J. Sánchez, B. J. Matuszewski, H. Chen, L. Yu, Q. Angermann, O. Romain, B. Rustad, I. Balasingham, K. Pogorelov, S. Choi, Q. Debard, L. Maier-Hein, S. Speidel, D. Stoyanov, P. Brandao, H. Córdova, C. Sánchez-Montes, S. R. Gurudu, G. Fernández-Esparrach, X. Dray, J. Liang, and A. Histace, "Comparative Validation of Polyp Detection Methods in Video Colonoscopy: Results From the MICCAI 2015 Endoscopic Vision Challenge," IEEE Transactions on Medical Imaging, vol. 36, pp. 1231-1249, June 2017.

[4] Y. Iwahori, T. Shinohara, A. Hattori, R. J. Woodham, S. Fukui, M. K. Bhuyan, and K. Kasugai, "Automatic polyp detection in endoscope images using a hessian filter.," in MVA, pp. 21-24, 2013.

[5] S. Y. Park and D. Sargent, "Colonoscopic polyp detection using convolutional neural networks," in Medical Imaging 2016: Computer-Aided Diagnosis, vol. 9785, p. 978528, International Society for Optics and Photonics, 2016.

[6] S. Ameling, S. Wirth, D. Paulus, and F. Vilarino, "Texture-based polyp detection in colonoscopy," in Bildverarbeitung für die Medizin, pp. 346350, Springer, 2009.

[7] C. Orlando, P. Andrea, D. Xavier, and B. Granado, "Polyps recognition using fuzzy trees," in Biomedical \& Health Informatics (BHI), 2017 IEEE EMBS International Conference on, pp. 9-12, IEEE, 2017.

[8] C. Orlando, P. Andrea, M. Christophel, D. Xavier, and B. Granado, "FPGA-Based Real Time Embedded Hough Transform Architecture for Circles Detection," in 2018 Conference on Design and Architectures for Signal and Image Processing (DASIP), pp. 31-36, Oct. 2018.

[9] O. Chuquimia, A. Pinna, X. Dray, and B. Granado, "Fpga-based real time embedded hough transform architecture for circles detection," in DASIP 2018-Conference on Design and Architectures for Signal and Image Processing, 2018.

[10] R. M. Haralick, "Statistical and structural approaches to texture," Proceedings of the IEEE, vol. 67, no. 5, pp. 786-804, 1979.

[11] O. Romain, A. Histace, J. Silva, J. Ayoub, B. Granado, A. Pinna, X. Dray, and P. Marteau, "Towards a multimodal wireless video capsule for detection of colonic polyps as prevention of colorectal cancer," in Bioinformatics and Bioengineering (BIBE), 2013 IEEE 13th International Conference on, pp. 1-6, IEEE, 2013.

\footnotetext{
${ }^{1}$ Thanks to Hana Raffoul for her corrections supported in this paper.
} 